\title{
Chronic Morphine Administration Delays Wound Healing by Inhibiting Immune Cell Recruitment to the Wound Site
}

Josephine L. Martin, ${ }^{*}$ Lisa Koodie, ${ }^{\dagger}$

Anitha G. Krishnan, ${ }^{\dagger}$ Richard Charboneau, ${ }^{\ddagger}$

Roderick A. Barke, ${ }^{\ddagger}$ and Sabita Roy ${ }^{\star \dagger}$

From the Department of Pharmacology, University of Minnesota, Minneapolis, Minnesota; the Department of Surgery, ${ }^{\dagger}$ Division of Basic and Translational Research, Minneapolis, Minnesota; and the Department of Surgery, ${ }^{\ddagger}$ Veterans Affairs Medical Center, Minneapolis, Minnesota

Patients prescribed morphine for the management of chronic pain, and chronic heroin abusers, often present with complications such as increased susceptibility to opportunistic infections and inadequate healing of wounds. We investigated the effect of morphine on wound-healing events in the presence of an infection in an in vivo murine model that mimics the clinical manifestations seen in opioid user and abuser populations. We show for the first time that in the presence of an inflammatory inducer, lipopolysaccharide, chronic morphine treatment results in a marked decrease in wound closure, compromised wound integrity, and increased bacterial sepsis. Morphine treatment resulted in a significant delay and reduction in both neutrophil and macrophage recruitment to the wound site. The delay and reduction in neutrophil reduction was attributed to altered early expression of keratinocyte derived cytokine and was independent of macrophage inflammatory protein 2 expression, whereas suppression of macrophage infiltration was attributed to suppressed levels of the potent macrophage chemoattractant monocyte chemotactic protein-1. When the effects of chronic morphine on later wound healing events were investigated, a significant suppression in angiogenesis and myofibroblast recruitment were observed in animals that received chronic morphine administration. Taken together, our findings indicate that morphine treatment results in a delay in the recruitment of cellular events following wounding, resulting in a lack of bacterial clearance and delayed wound closure. (Am J Pathol 2010, 176:786-799; DOI: 10.2353/ajpath.2010.090457)
Morphine, a mu opioid receptor agonist, is a well-documented analgesic derived from the extracts of opium poppy plants (Papaver somniferum). On binding to its receptors located in the central nervous system, morphine mimics the actions of its endogenous peptide neurotransmitter, $\beta$-endorphin, to inhibit nociceptive stimuli responsible for pain perception. A comparison of the distribution patterns of opioid receptors in the human brain and that reported for the rodents suggests a homologous expression pattern in many regions, especially those that relate to pain and analgesia. ${ }^{1-3}$ Furthermore, most leukocytes (monocytes, neutrophils, and $T$ and $B$ lymphocytes) express low levels of mu-opioid receptors and opioid peptides, with a similar pattern of distribution in humans and rodents and opioid receptor levels are markedly induced on activation. ${ }^{4,5}$ Although chronic morphine administration has become the gold standard in managing long term pain, it has unwanted side effects, such as respiratory depression, suppression of gastrointestinal motility, and immunosuppression, through its binding to mu opioid receptors on cells in the brainstem, in the gastrointestinal, and on immune cells, poses user complications. $^{6-11}$ Several studies have also documented the process by which wound healing occurs. ${ }^{12-20} \mathrm{How}$ ever, the underlying mechanisms why wound healing complications are frequently present in populations that have chronic morphine administered clinically or in the drug abusing population, have not been fully explored.

Wound healing is an intricately regulated sequence of cellular and biochemical events orchestrated to restore

Supported by grants R01 DA12104, R01 DA DA022935, K02 DA015349, and P50 DA11806 (S.R.) from The National Institutes of Health and funds from the Minneapolis Veterans Affairs Medical Center (R.A.B.).

Accepted for publication October 21, 2009.

J.L.M. is currently employed at Medtronic, Incorporated. Research conducted for this study was completed prior to employment at Medtronic, Inc. No conflict of interest is affiliated with this research.

Supplemental material for this article can be found on http://ajp. amjpathol.org

Address reprint requests to Sabita Roy, Ph.D., Department of Pharmacology, University of Minnesota, 6-120 Jackson Hall, 321 Church Street SE, Minneapolis, Minnesota, 55455. E-mail: royxx001@umn.edu. 
tissue integrity after injury. The sequence of events in wound healing process begins immediately after injury and occurs in two phases: a pro-inflammatory phase, which are needed to ensure adequate bacterial clearance at the site of tissue injury, and a re-epithelialization and neovascularization phase ${ }^{12-20}$ to ensure proper wound closure..$^{21}$ It is important to note that resolution of bacterial clearance is essential in order for the wound closure processes to take place.

Varying populations of cells migrate to the site of the wound in a sequential fashion following wounding. Platelets are the initial responders post injury, followed by neutrophils, macrophages, lymphocytes, and fibroblast cells. Platelets, peaking 12 hours post-wounding, are required for coagulatory events, whereas neutrophils and macrophages (peaking on days 1 and 3 , respectively) are pro-inflammatory populations key in migratory and proliferative events. The phagocytic neutrophils and macrophages are key players in recognizing and eradicating pathogens. During the innate immune response, neutrophils mainly elicit their anti-pathogenic effects by engulfing and killing pathogens via reactive oxidative mechanisms. ${ }^{22}$ Macrophages are then recruited for further bactericidal action against pathogens, as well as engulfment of neutrophil debris. In addition to their individual contributions to wound healing, each cell type has been found to produce and secrete potent chemotactic factors that enable the migration and activation of subsequent cell populations.

Chemokines and cytokines, such as keratinocyte-derived cytokine $(\mathrm{KC})$, macrophage inflammatory protein 2 (MIP-2), and monocyte chemotactic protein-1 (MCP-1), play critical roles in regulating the inflammatory immune response following tissue injury or infection. Injured epithelial cells, and resident pro-inflammatory cells, such as platelets, produce, secrete, and initiate a chemical gradient to activate and attract leukocytes to the area of injury. During this time, potent neuropeptides are also released in the peripheral damaged tissues that bind to their receptors, located on endothelial and immune cells promoting leukocyte adhesion and translocation. ${ }^{16-23}$ As a result, circulating activated immune cells readily cross the vascular compartment at the site of injury and migrate toward the chemical gradient. The primary role of recruited leukocytes, including neutrophils and macrophages, is to initiate tissue debridement and to launch a pro-inflammatory response to protect against opportunistic invasion of foreign pathogens.

The clearance of the debris and infectious organisms promotes resolution of the inflammatory phase and initiation of the repair phase, which includes formation of granulation, re-epithelialization, and neovascularization (angiogenesis). Fibrin matrices, collagen deposits, and the formation of new blood vessels are all events that promote wound closure processes. New blood vessel formation occurs 7 days post-wounding. Angiogenic processes are measured based on vessel formation, vessel branching, and vessel length. Potent pro-angiogenic factors including vascular endothelial growth factor (VEGF) $)^{21,24}$ and hypoxia inducible factor- $1 \alpha^{25}$ act on promoting these angiogenic events at the translational and transcriptional level.

Clinically, the prevalence of non-healing wounds among the heroin-addicted population has also been well documented. Non-healing wounds, at the site of drug injection, in this population have been attributed to unclean practices such as needle sharing. However, recent in vitro and ex vivo studies indicate that compromised immune function within this abusing population may be the reason behind the delay in the wound healing process. ${ }^{26}$ Although wound healing mechanisms are well established in the literature, these studies have been performed under sterile conditions. Such analysis would have translational implications for surgical and postsurgical practices. The fact that over $90 \%$ of all cutaneous wounds incurred in nature are likely to be infected, the need to study the infection component on wound healing processes is essential.

This study is the first to standardize and characterize a murine in vivo model that mimics the clinical presentation of wound healing complications seen in opioid user and abuser populations. With the use of this model, we addressed the following questions: 1) Does chronic morphine administration modulate the wound healing process in the presence of an infection? 2) At what level(s) does morphine act on the innate pro-inflammatory phase of wound healing? 3) Does morphine modulate later wound healing events such as angiogenesis and myofibroblast induction?

\section{Materials and Methods}

\section{Animals and Experimental Design}

A model for studying cutaneous wound healing was used to determine immune cell migratory patterns to the wound, chemokine gradient profiles, and wound closure rates in mice. One hundred and ninety-two C57BL/6J male mice (Jackson Laboratories, Bar Harbor, ME) at approximately 6 to 7 weeks of age and 20 to $25 \mathrm{~g}$ body weights were randomly assigned to one of five treatment groups. Mice were injected i.p. with either saline or morphine sulfate to prevent multiple wounding and to simulate chronic morphine use conditions $(6.25 \mathrm{mg} / \mathrm{kg}$ morphine every 6 hours for a total of $25 \mathrm{mg} / \mathrm{kg}$ morphine over 24 hours). This dose of morphine results in plasma levels of morphine in the 150 to $200 \mathrm{ng} / \mathrm{ml}$ range at steady state, which is well within the range observed in patients receiving morphine for analgesic purposes for moderate to severe pain (20 $\mathrm{ng}$ to $2000 \mathrm{ng} / \mathrm{ml}$ ) and in heroin abusers (200 ng to $2000 \mathrm{ng} / \mathrm{ml}$ ). ${ }^{27-31}$ On day 0, mice were anesthetized with ketamine (65 mg/kg body weight) and $x y-$ lazine (5.5 mg/kg body weight) and implanted with either a placebo or morphine $(75 \mathrm{mg}$ ) (National Institute on Drug Abuse, Bethesda, MD) slow release pellet s.c., in the shaved region to the left of the dorsal midline, the incision was closed with a 9-mm wound autoclip (Becton Dickinson, Sparks, MD). Serum morphine concentrations following pellet implantation have steady-state dose concentrations in the range of 250 to $400 \mathrm{ng} / \mathrm{ml} .^{32} \mathrm{~A}$ deep 
subcutaneous pocket was made just above the right hind limb to accommodate a polyvinyl alcohol (PVA) sponge $(5 \mathrm{~mm} \times 5 \mathrm{~mm})$ and an 8-mm diameter full-thickness skin excision was made just above the left hind limb.

Animals were housed four mice per cage according to treatment groups under controlled 12:12 light/dark cycles and controlled temperatures $\left(25^{\circ} \mathrm{C}\right)$. Animals received standard food and tap water freely accessible from cages. Mice subjected to placebo or morphine pellet implantation according to the protocols and models previously described, were returned to their cages and separated by experimental group classifications: (i) saline i.p. + placebo pellet + saline sponge implant; (ii) saline i.p. + placebo pellet + LPS sponge implant; (iii) morphine sulfate i.p. + morphine pellet + saline sponge implant; (iv) morphine sulfate i.p.+ morphine pellet + LPS sponge implant; and (v) morphine sulfate and naloxone i.p. + morphine pellet and naltrexone pellets + LPS sponge implant.

Discomfort, stress, and injury to vertebrate subjects were minimized. The Institutional Animal Care and Use Committee at the University of Minnesota approved all protocols and surgical procedures in these studies in agreement with the guidelines set forth by the National Institute of Health Guide for Care and Use of Laboratory Animals.

\section{Morphine, Naloxone, and Lipopolysaccharide Preparation}

Morphine sulfate (National Institute on Drug Abuse, Bethesda, MD) was reconstituted in $1 \times$ Hanks' Buffer Saline Solution (HBSS) to a $1 \mu \mathrm{mol} / \mathrm{L}$ concentration. Placebo, morphine, and naltrexone pellets were generously provided by the National Institute on Drug Abuse. Naloxone preparation was in $1 \times$ HBSS at a final concentration of 10 $\mu \mathrm{mol} / \mathrm{L}$. Lipopolysaccharide (LPS) was reconstituted to yield a $1 \mu \mathrm{g} / \mathrm{ml}$ concentration in $1 \times$ HBSS.

\section{H\&E Staining of Wound Tissues}

Cutaneous wound bed tissues were excised on days $0.5,1$, 2 , 3, or 4 using surgical forceps and scissors. Whole wounds were placed into labeled tissue cassettes and fixed in 10\% formalin, $4 \%$ paraformaldehyde solution (Sigma, St. Louis, MO). Samples were sent to the University of Minnesota Fairview-Riverside Hospital's pathology laboratory for paraffin embedding and staining. Parallel microtome sections were analyzed using immunocytochemistry.

\section{PVA Sponge Preparation, Enzyme-Linked Immunosorbent Assay, and Fluorescence-Activated Cell Sorting}

Saline- or LPS- [1 mg/ml] moistened PVA sponges (Micronova, Torrance, CA) were cut into $5 \mathrm{~mm} \times 5 \mathrm{~mm}$ squares and implanted just above the right hind limb. On removal on designated days, sponges saturated in $1 \mathrm{ml}$ of $1 \times$ HBSS were placed in a $12 \mathrm{cc}$ syringe to extract cells. Cells were centrifuged at $3000 \mathrm{rpm}$ (1000 rcf) for 5 minutes at $4^{\circ} \mathrm{C}$. Supernatants were transferred to fresh 1 $\mathrm{ml}$ Eppendorf tubes and underwent a second centrifugation step (3000 rpm for 3 minutes) to remove any debris. Supernatants were analyzed for MIP-2, KC, and MCP-1 enzyme-linked immunosorbent assay (ELISA) protein analysis (R\&D Systems, Minneapolis, MN).

Pellets, containing cells that migrated to the PVA sponges, were re-suspended in $1 \mathrm{ml}$ of $1 \times$ HBSS and counted (trypan blue exclusion). Then, $1 \times 10^{7}$ cells per sample were aliquoted into round-bottom polystyrene tubes (BD Falcon, Bedford, MA), labeled using LyG-6 and F4/80 surface markers (1:1000 and 1:500 dilutions, respectively, BD Bioscience, San Jose, CA) according to standard protocol and processed for Flow Cytometry FACScan analysis (Beckman Dickerson, Parsippany, NJ) according to CELLQwest acquisition and analysis protocols.

\section{Wound Excising, Imaging, and Analysis}

Cutaneous wounds were inflicted above the left hind limb with the use of surgical forceps and scissors. Excisions followed an 8-mm diameter biopsy punch template placed on top of the hind limb and traced in permanent marker. Wounds were photographed 24 hours later using a Bencher Illuma System light control photography mounting stage (Fryer Co, Huntley, IL) and a digital camera (Kodak C513, Rochester, NY). At varying time points, wounds were photographed using the same settings as used initially. Percentages of wound closure events were calculated as = [1 - (wound pixel area on end point day/wound pixel area on day 1) $\times 100 \%]$. Wound pixel areas were determined using Adobe Photoshop software.

\section{Isolation of Bone Marrow-Derived Neutrophils}

Bone marrow neutrophils were isolated as described by Deshmukh et al. ${ }^{33}$ Briefly, the femurs and tibias were removed and stripped of all muscle. The marrow was flushed from each bone with HBSS, and the cell aggregates were disrupted by trituration to generate a unicellular suspension. The cells were pelleted, and erythrocytes were removed by hypotonic lysis. Neutrophils were isolated from the remaining cells by centrifugation over discontinuous Percoll (Amersham Biosciences) gradients at $500 \times g$ for 30 minutes at $4^{\circ} \mathrm{C}$, consisting of $55 \%(\mathrm{v} / \mathrm{v})$, $65 \%(\mathrm{v} / \mathrm{v})$, and $75 \%(\mathrm{v} / \mathrm{v})$ Percoll in PBS. Neutrophils were recovered at the interface of the $65 \%$ and $75 \%$ fractions and subjected to cytospin and Wright-Giemsa staining. The purity of neutrophil in the sample was determined to be greater than $90 \%$ pure in the neutrophil-rich fraction as determined by fluorescence-activated cell sorting (FACS) analysis (Ly6G staining) and Wright-Giemsa staining. Viability was determined to be $95 \%$ using trypan blue staining.

\section{High-Throughput Screen Transwell Permeable Chemotaxis Chamber Analysis}

Chemotaxis assays were performed using a 96-well plate with 3.0- $\mu \mathrm{m}$ polycarbonate membrane inserts (Corning 
Inc., Corning, NY). Bone marrow was harvested from green fluorescent protein (GFP)-transgenic mice and treated in vitro with either saline or morphine for 24 hours. Neutrophils were purified using density centrifugation as described above and purity was verified using FACS analysis, using the neutrophil-specific surface marker Ly6G. Then, $5 \times 10^{6}$ neutrophils were placed in the top chamber of the chemotaxis plate in quadruplicate. Day-1 PVA sponge supernatants from placebo- and morphinetreated mice were placed in the bottom chambers. The chemotaxis chamber was incubated at $37^{\circ} \mathrm{C}, 5 \% \mathrm{O}_{2}$ for 1 hour. Independent quantification of top and bottom chambers was performed using an Omega FLUOstar fluorescence microplate reader (BMG LABTECH Inc, Durham, NC). GFP was detected at a 485:520 excitation/emission wavelength ratio.

\section{Matrigel Plug Preparation}

Sponge fluids (4 day) isolated from placebo + LPS- and morphine + LPS-treated animals were resuspended in Matrigel solution (200 $\mu$ l to $500 \mu \mathrm{l}$ Matrigel; BD Bioscience, San Jose, CA) and injected s.c. above the hind limb. Matrigel reacts with the body temperatures of mice to form a noninvasive plug. Seven days post-Matrigel injection, gross morphology and vascular invasion were evaluated as described previously. ${ }^{34}$ Mice were euthanized before the removal of the Matrigel plug.

Matrigel plugs were snap frozen in liquid nitrogen and cryostat-sectioned. Eight-micron-thick sections of the plugs were evaluated using $\times 100$ microscopy. Sections were fixed and incubated with an anti-CD31-phycoerythrin (PE) monoclonal antibody. Quantitative analysis of vessel number, vessel branching, and vessel length were measured. Images of stained sections were captured using the Olympus BX60 Upright microscope and analyzed using the Photoshop Imaging Processing Tool kit.

\section{Determination of Bacterial Dissemination into Blood, Liver, and Spleen}

Mice were administered saline or morphine i.p. 24 hours before implantation of pellets and PVA sponge (day -1). On day 0 , mice underwent implantations of placebo or morphine slow-release pellets as described above. PVA sponges containing GFP-tagged Escherichia coli [kanamycin-insensitive (colony forming units $=18$ )] were implanted and the wound excised as described above. On day 7 , spleens and livers were harvested, and blood was drawn via cardiac puncture. ${ }^{35}$ Spleen and liver tissues were homogenized in $0.5 \mathrm{ml}$ of $1 \times$ HBSS. Plating of tissues onto sheep blood agar plates (500 $\mu$ l/plate) containing kanamycin were performed using aseptic technique with a glass spreader. Serially diluted heparinized blood samples were also plated onto blood agar plates. Plates were incubated at $37^{\circ} \mathrm{C}, 5 \% \mathrm{CO}_{2}$ overnight. E. coli GFP-positive colonies grown on plates were counted manually.

\section{Statistical Analysis}

Unpaired student $t$-test was used to test for significant differences in leukocyte numbers between morphine and control treatment groups at the various time points. Data are expressed as the mean \pm SEM. Differences were considered significant when $P \leq 0.05$. Each experiment was performed in triplicate unless otherwise stated. Statistical computer packages STATVIEW (SAS Institute, Cary, NC) and SPSS (Chicago, IL) were used.

\section{Results}

\section{Effects of Morphine on Wound Closure and Wound Integrity Following Tissue Injury}

To determine the effect of chronic morphine on wound healing, we initially measured wound closure at different days following wounding. Wound beds of morphinetreated mice in the presence or absence of LPS were compared with placebo and naltrexone groups (Figure 1, $A$ and $B$ ). Mice treated with either morphine alone or morphine in the presence of LPS showed significantly delayed wound closure $\left({ }^{*} P \leq 0.05\right)$ with a marked presence of tissue necrosis, pus, and edema/swelling, indicative of insufficient bacterial clearance and sustained inflammation, when compared with either placebotreated or placebo + LPS-treated mice. Wounds of mice that received co-administration of naltrexone (an opioid antagonist) presented similar wound morphologies to placebo treated groups. To determine wound closure quantitatively, digitized pictures of wounds were scanned and analyzed. Wound sizes at any given time point after wounding were expressed as percentage of initial (day 0 ) wound area for placebo- and morphine-treated mice. Results are expressed as the mean \pm SE $(n=6, P<$ $0.05)$. Wound closure was significantly delayed in the morphine + LPS treatment group, when compared with the placebo + LPS group at every time period tested (Figure $1 \mathrm{C}$ ). To further determine the role of the muopioid receptor in morphine's effect, wound closure following wounding was performed in the MORKO (muopioid receptor knock-out) mice. Although wound closure was significantly delayed in the morphine-treated wildtype mice, wound closure in the morphine-treated MORKO mice was not visibly different from the wild-type placebo-treated (Figure 1, C and D) or MORKO placebotreated mice (data not shown). These findings clearly establish that chronic morphine treatment significantly inhibits wound healing, similar to what is reported in the opioid use and abuse populations and therefore is a useful model to investigate the mechanisms underlying the inhibitory role of morphine in wound healing events. These data also implicate the Mu-opioid receptors in mediating morphine's effect on wound closure since morphine-induced inhibition of wound closure was completely abolished in the Mu-opioid receptor knock-out mice. To further explore the mechanism by which chronic morphine suppresses wound healing processes, we in- 


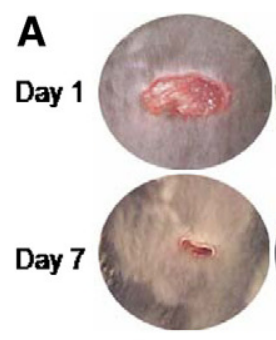

Placebo

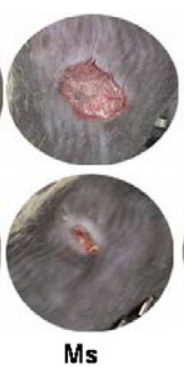

Ms

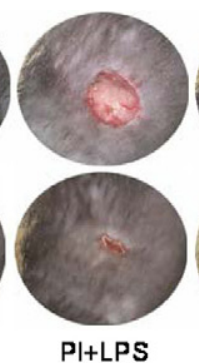

PI+LPS

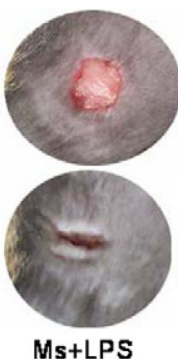

Ms+LPS

C

${ }_{120}$

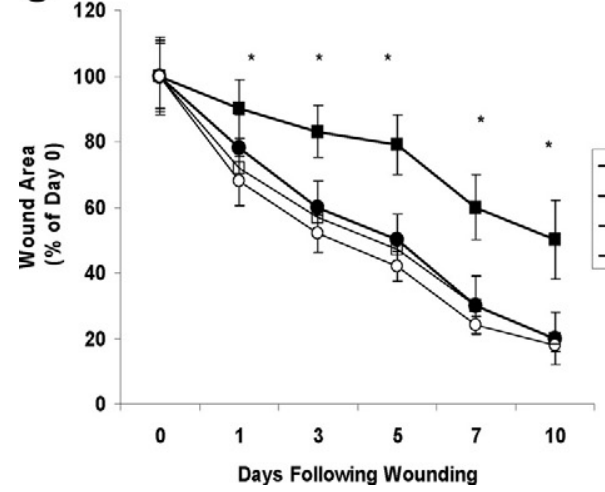

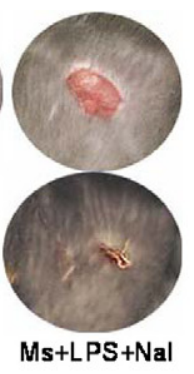

D

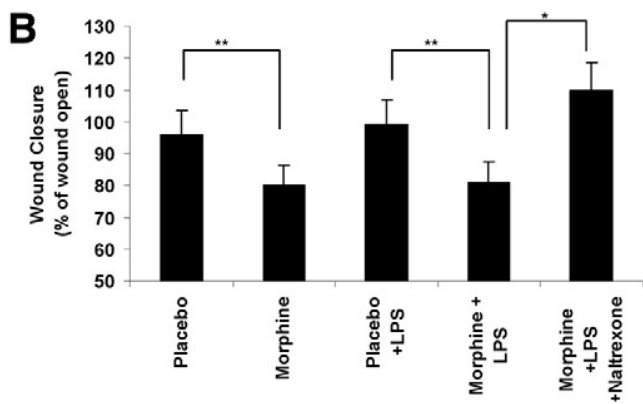

Figure 1. A: Gross morphology of cutaneous wounds in hind limbs of mice. Cutaneous wounds of mice treated with placebo, morphine, or morphine plus naltrexone in the presence or absence of LPS were photographed on days 1 and 7 . Ratios of quantified pixel areas of the wounds were used in determining the rate of wound closure. A marked decrease in wound healing integrity was seen in the morphine-treated groups. Integrity is based on the quality of healing. Wounds of morphine-treated animals presented with increased necrotic tissue, pus, and marked edema/swelling. Co-administration of naltrexone reversed the effects seen in the morphine-treated groups. Following chronic morphine administration, in the presence and absence of LPS, wounds showed a significant decrease in closure rates compared with control groups. B: Quantified analysis of pixel area wound closure events. Percentages of wound areas were quantified to measure closure rates between treatment groups. Areas of wound beds from mice treated with morphine showed significant delay in closure when compared with their respective control groups. Co-administration of naltrexone restored the morphine-mediated delay in wound closure to rates seen in the placebo treated group. Significance of $P$ values are ${ }^{*} P \leq 0.001,{ }^{* *} P \leq 0.01(n=4)$. C: Temporal decrease in wound closure in morphine-treated animals. Wounds were photographed on days $0,1,3,5,7$, and 10 following wounding. Quantified pixel areas of the wound were calculated as percentage of wound area at day 0 . Wound closure measured at each time points were significantly lower in the morphine-treated wild-type (WT) mice when compared with all other treatment groups. * $P<$ $0.05(n=4)$. Wound closure rate of morphine-treated MORKO mice were similar to wild-type placebo control. D: Morphine-induced inhibition in wound closure was abolished in MORKO mice. Wild-type and MORKO mice were treated with morphine or placebo pellet and wound beds photographed at day 7 . Wound closure in morphine-treated MORKO mice was similar to wild-type placebo-treated animals.

vestigated the migratory patterns of pro-inflammatory leukocytes into wound tissues.

\section{Migration Patterns of Leukocyte Infiltration into Wound Beds Following Morphine Treatment}

To determine the infiltration patterns of neutrophils and macrophages, we investigated early wound healing time points where both populations are known to peak. Wound bed tissues excised on days $0.5,1,2,3$, or 4 were fixed in $10 \%$ formalin, mounted in paraffin, microtome-sectioned, and stained with H\&E (see Supplemental Figure $\mathrm{S} 1$ at $h$ ttp://ajp.amjpathol.org and Figure 2A). Representative slides were photographed using the Zeiss Axiovert Upright Microscope ( $\times 10$ magnification). As early as 12 hours post-injury, the presence of granular multilobular neutrophils was seen in placebo-treated mice (Supplementary Figure S1, at http://ajp.amjpathol.org), and by day 4 , a robust migration of neutrophils out of the blood vessels into the surrounding tissues was observed in the placebo + LPS treated mice (Figure 2, A and B). However, this migratory trend was significantly suppressed when morphine was administered with fewer leukocytes observed in the parenchyma (Supplementary Figure S1, at http://ajp.amjpathol.org, Figure 2, A and B). In the placebo group there was clear migration of neutrophils out of the surrounding blood vessel into the tissue area (Figure 2B). In contrast in the morphine-treated animals, even in the presence of the potent pro-inflammatory stimulus, LPS, leukocytes failed to adequately migrate in response to the endotoxin and remained in the blood vessel (Figure 2B). These studies clearly indicate that chronic morphine administration significantly suppresses leukocyte infiltration in response to tissue injury, even in the presence of the potent pro-inflammatory stimulus, LPS.

To determine whether morphine treatment results in a delay in later wound healing and repair events such as angiogenesis and granulation, wounds of animals at day 7 were analyzed for CD-31 (angiogenesis) and $\alpha$-smooth muscle cell (SMC) staining (myofibroblast). Angiogenesis and myofibroblast content of wounds in 7-day wounds of placebo- and morphine-treated animals were determined using two sections from each wound $(n=5)$ and analyzed for CD31 (PE) and $\alpha$ SMC (fluorescein isothiocyanate) histological localization. Sections were scanned at low magnification $(\times 10)$ to identify hot spots. Areas of greatest vessel and myofibroblast density were then examined under higher magnification $(\times 20)$ and counted. 


\section{A Placebo +LPS (DAY 4)}
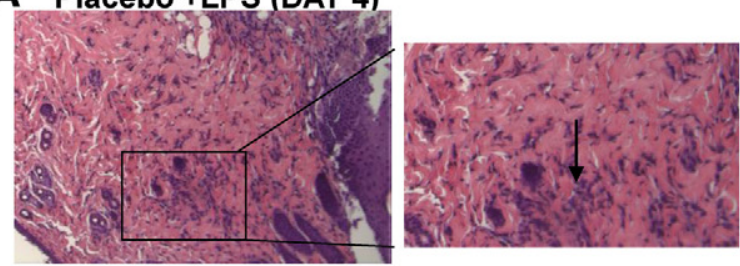

Morphine + LPS (DAY 4)

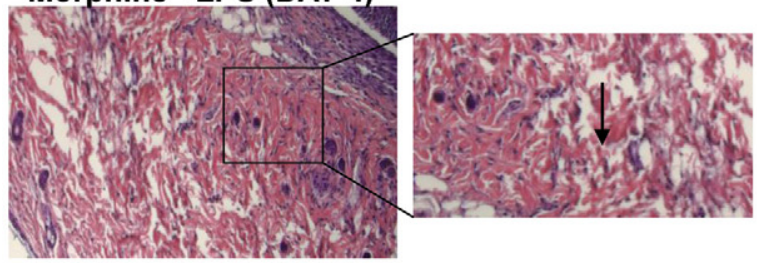

C

Placebo +LPS (day 7)
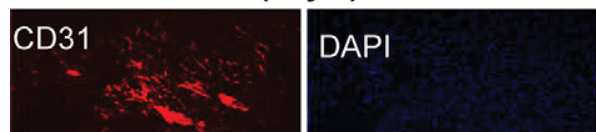

Morphine +LPS (day 7)
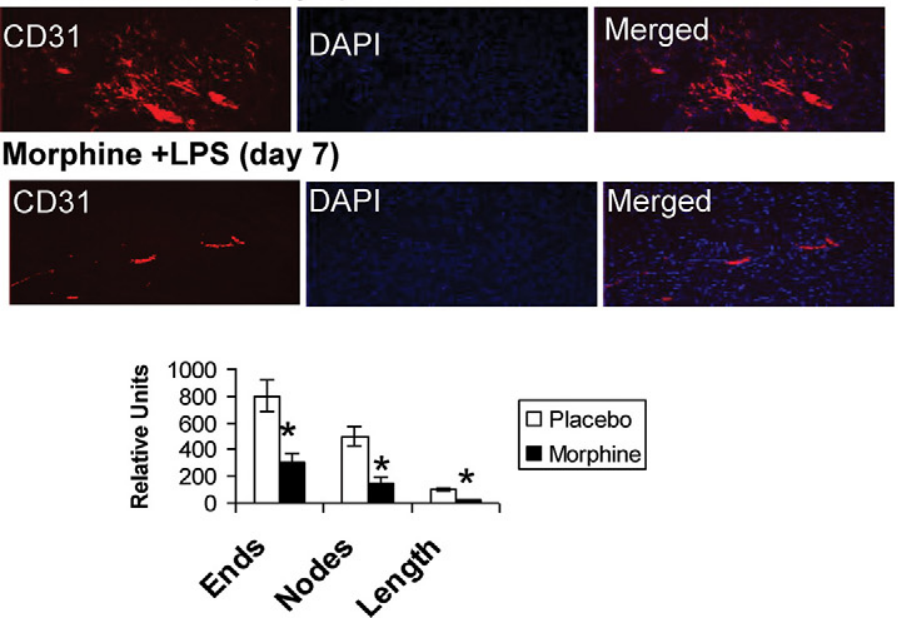

B
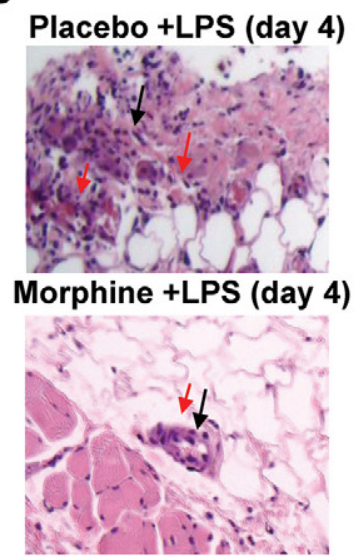

D Placebo + LPS (day 7)
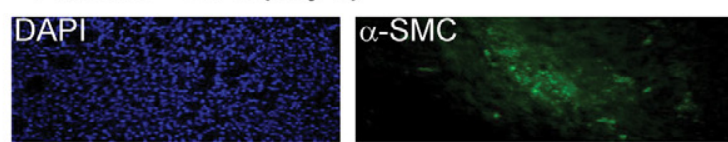

Morphine + LPS (day 7)
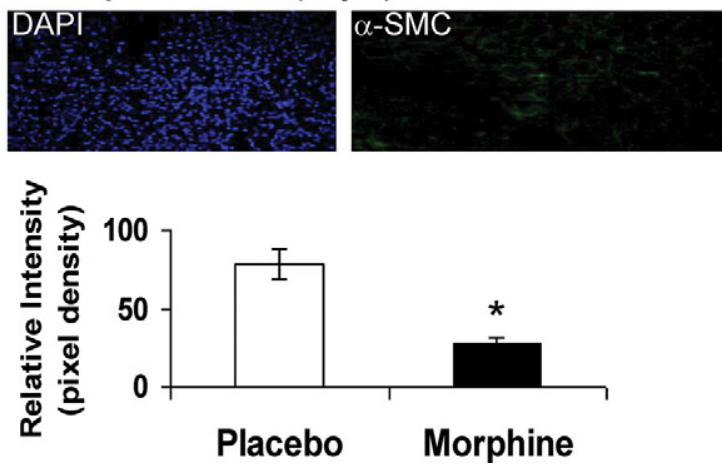

Figure 2. A: Morphine delays leukocyte migration into wound tissue beds. Histological analysis of wound beds taken from the hind limbs was performed using H\&E staining techniques. Representative wound sections show significant reduction of infiltrating leukocytes (shown with black arrow) into wound tissues of mice treated with morphine 4 days post-injury. B: Wound sections of morphine-treated animals show significantly lower transmigration of leukocytes out of blood vessels into wound beds. Red arrows indicate blood vessels and black arrows infiltrating leukocytes. C: Representative CD31 staining of wound sections was performed on day seven wound sections. Eight-micron, paraffin-embedded sections were stained with anti-CD 31-PE antibody, an endothelial marker. Cell number was determined using 4,6-diamidino-2-phenylindole staining. CD-31 staining was markedly reduced in morphine-treated wounds ( $n=4$ ). Quantitative representation of vessel density (ends), vessel branching (nodes) and vessel length (length) show significant reduction in all parameters tested in morphine-treated animals. ${ }^{*} P<0.01(n=4)$. D: Representative $\alpha$-SMC staining was performed on day seven wound sections. 8 micron paraffin embedded sections were stained with anti $\alpha$-SMC-fluorescein isothiocyanate antibody, a myofibroblast marker. Cell number was determined using 4,6-diamidino-2-phenylindole staining. $\alpha$-SMC-fluorescein isothiocyanate staining was markedly reduced in morphine-treated wounds $(n=4)$. Quantitative evaluation showed significant reduction in $\alpha$-SMC expression in morphine-treated wounds. ${ }^{*} P<0.01(n=4)$.

CD-31 fluorescent images were binearized for PE-positive pixels, and skeletonized (gray images) using a Reindeer Image Analysis tool kit and Adobe PhotoShop as previously described by Wild et al. ${ }^{34}$ This method of analysis allows quantification of microvessel density based on average blood vessel numbers (ends, E), blood vessel branching (nodes, F), and blood vessel length (G). The statistical differences are based on comparisons between means using a Student's t-test, and significance was set at $P<0.05$. Wounds of placebo treated animals showed a significantly greater CD-31 staining with a greater blood vessel number, branch points, and length when compared with 7-day wounds of morphine-treated animals (Figure 2C). Seven-day wound of placebotreated animals also showed significant $\alpha$ SMC staining (Figure 2D), when compared with that observed in morphine-treated animals (Figure 2D). These results clearly demonstrate that morphine treatment results in a dramatic decrease in early wound recruitment of leukocytes that may contribute to the delay in the initiation of later repair events such as re-epithelialization, granulation, and angiogenesis.

\section{Neutrophil and Macrophage Infiltration into PVA Sponge Implants Following Morphine Treatment}

To further determine whether morphine modulates leukocyte recruitment to wound sites, mice were treated with either morphine or placebo as described above. PVA sponges were soaked in either saline or LPS $(1 \mu \mathrm{g} / \mathrm{ml})$ and inserted into pockets as a stack. At days 0.5, 1, 2, 3, 4 , and 5, sponges were removed, placed in a $3 \mathrm{ml}$ syringe barrel and centrifuged in a $50 \mathrm{ml}$ centrifuge tube 


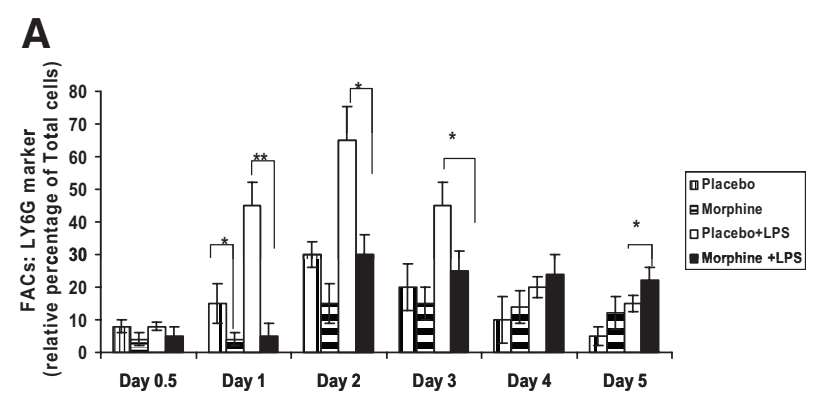

B

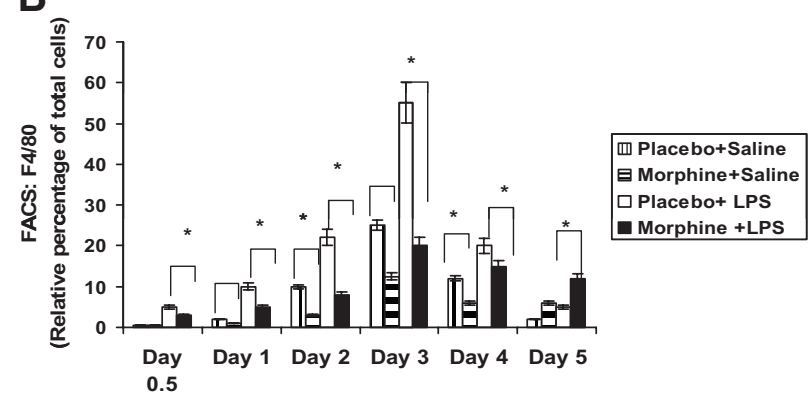

Figure 3. A: FACS analysis of LY6G-labeled neutrophils from PVA sponges. Following injury and removal of PVA sponges, cells were extracted and counted using trypan blue exclusion. $1 \times 10^{7}$ cells were placed into each FACS tubes, labeled with LY6G mouse antibodies, and quantified. In the presence of LPS, a marked increase in cells positive for LY6G (neutrophils) migrated into the PVA sponge 24 hours post-injury. However, significantly fewer LY6G-positive cells were detected when morphine was co-administered and this cell population and did not peak until 72 hours post-injury. Significance of $P$ value is ${ }^{* *} P \leq 0.01,{ }^{*} P<0.05(n=4)$. B: FACS analysis of F480-labeled macrophages from PVA sponges. Following injury and removal of PVA sponges, $1 \times 10^{7}$ cells were placed into each FACS tubes, labeled with $\mathrm{F} 4 / 80$ mouse antibodies, and quantified. A significant decrease ( ${ }^{*} P \leq$ $0.01, n=4$ ) in F4/80-positive cells (macrophages) was seen following co-treatment of morphine and LPS on days $0.5,1,2,3$, and 4 . Peak recruitment on day 3 post-injury, was also significantly suppressed ( ${ }^{*} P \leq 0.01, n=$ $4)$ in the morphine + LPS group. Sustained and significant recruitment was observed in the morphine-treated group on day $5\left({ }^{*} P \leq 0.01, n=4\right)$.

for 15 minutes at 1,200 rpm. Cells and supernatant were collected. Cells were treated with antibody specific for neutrophil (LyG-6/Gr-1-fluorescein isothiocyanate BD Pharmingen) and macrophage markers (F4/80-PE, clone Cl:A3-1), neutrophils, and macrophages were quantified using flow cytometry. Neutrophil recruitment began at day 1 and peaked at day 2 in both the placebo-saline and placebo-LPS groups (Figure 3A). As expected the neutrophil recruitment pattern in the placebo-LPS group was significantly greater than the placebo-saline group at both day 1 and day 2 . In contrast, there was a dramatic delay in neutrophil recruitment in the morphine + saline and morphine + LPS group, as compared with its control. The peak recruitment of neutrophils in the morphine + saline group and morphine + LPS group was also significantly lower than the placebo + saline and placebo + LPS groups. Interestingly, while neutrophil numbers returned to base line levels in the placebo-treated groups, a sustained recruitment of neutrophils was observed at day 5 in the morphine-treated groups.

When evaluating the effects of morphine on macrophage recruitment patterns, a similar profile was observed as with neutrophils recruitment (Figure 3B). On day 1 post-injury, the migratory pattern of macrophages stained with surface marker F4/80 was significantly in- creased in response to LPS (placebo + LPS). However, macrophages failed to migrate in response to LPS when morphine was co-administered (morphine + LPS). By day 3 , when macrophages are typically seen post-injury, significantly lower numbers of macrophages were detected when morphine was administered in the presence or absence of LPS. This suppression by morphine of LPS-induced macrophage recruitment continued as late as day 4 post-injury $\left({ }^{* *} P<0.01\right)$. At day 5 , macrophage numbers in the placebo + saline and placebo + LPS group returned to baseline level, however, in contrast, in the morphine + LPS group, a sustained recruitment of macrophages was observed indicating lack of bacterial clearance and sustained inflammation.

Taken together, these findings suggest that morphine plays a critical role as an immunosuppressor during the early phase of wound repair by 1) delaying neutrophil infiltration toward a LPS-mediated environment, and 2) consequently reducing macrophage infiltration. Delay in neutrophil and macrophage recruitment in the morphinetreated animals may be contributing to the delay in wound healing since sequential recruitment of immune cells at specific times following wounding contributes to later wound repair mechanisms.

\section{Effects of Secreted Protein Levels for Chemoattractants MIP-2, KC, and MCP-1 following Morphine Treatment}

Next, we explored whether the morphine-mediated 24hour delay in neutrophil migration was a result of an altered chemical gradient. To investigate this possibility, protein levels of the potent neutrophil (MIP-2 and KC) and macrophage (MCP-1) chemoattractants from the PVA sponge extracts were measured.

Supernatants collected on days 1 and 3 from PVA sponge extracts underwent MIP-2 (Figure 4A), KC (Figure $4 \mathrm{~B}$ ), and MCP-1 (Figure $4 \mathrm{C}$ ) quantikine ELISA protein detection analysis, respectively. At each time point, a significant elevation of MIP-2 (Figure 4A), KC (Figure 4B), and MCP-1 (Figure 4C) was seen when LPS was present. It was surprising to observe that morphine treatment did not result in any significant decrease in MIP-2 expression either at day 1 or at day 2, although a significant decrease in neutrophil recruitment was observed. When KC expression levels were measured, a significant increase in $\mathrm{KC}$ levels was observed at both days 1 and 3 in the placebo-treated LPS and placebo-treated saline groups. $\mathrm{KC}$ levels in the placebo group was significantly higher at day 1 compared with day 3 implicating a role for $\mathrm{KC}$ in the early recruitment of neutrophils following wounding. However, when KC levels were measured in the morphinetreated groups a significant decrease was observed at both day 1 and day 3 . These data suggest that the decrease in the expression of $\mathrm{KC}$ at the early phase of wounding may be a potential mechanism for the delay and decrease in neutrophil migration in the morphinetreated animals.

When the secretion levels of the macrophage chemoattractant MCP-1 were evaluated, it was found that on 
A
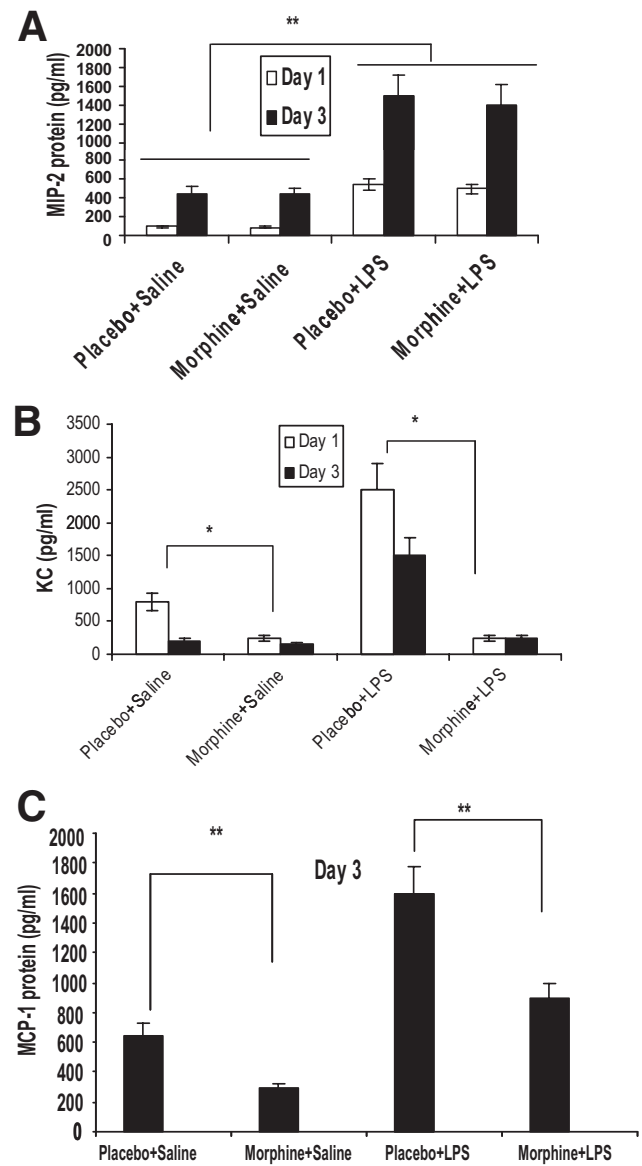

Figure 4. A: MIP-2 expression profile from PVA sponge extracts. The potent neutrophil chemoattractant MIP-2 from PVA sponge supernatants were analyzed using a MIP-2 protein detection ELISA kit. Interestingly, no significant modulation of protein expression was seen between placebo- and morphinetreated groups on days 1 and 3 post-injury. However, a significant ${ }^{* *} P<$ $0.01, n=4$ ) elevation of MIP-2 protein levels were seen in response to the endotoxin LPS (placebo and morphine in the presence of LPS). B: KC expression profiles from PVA sponge extracts. The potent neutrophil chemoattractant KC from PVA sponge supernatants were analyzed using the KC protein detection ELISA kit. Interestingly, $\mathrm{KC}$ levels in the placebo group were higher at day 1 compared with day 3 in both LPS treated and nontreated animals. Morphine treatment resulted in a significant $\left({ }^{*} P<0.01, n=\right.$ 4) reduction in $\mathrm{KC}$ protein levels, when compared with placebo-treated groups on both days 1 and 3 post-injury. A significant $\left({ }^{*} P<0.01, n=4\right)$ elevation of $\mathrm{KC}$ protein levels were seen in response to the endotoxin LPS. C: $\mathrm{MCP}-1$ expression profiles from PVA sponge extracts. The macrophage chemokine MCP-1 from PVA sponge supernatants was analyzed using ELISA By day 3 post-injury, significant suppression ( $\left.{ }^{* *} P \leq 0.01, n=4\right)$ of MCP-1 was seen following morphine administration when compared with its control (placebo). In response to the LPS stimuli, MCP-1 protein levels were markedly increased (placebo + LPS). However, a significant (*** $P \leq 0.01, n=4)$ decline in protein expression was seen when morphine was co-administered (morphine + LPS).

day 3 , in the presence of LPS, placebo-treated mice showed elevated expression of MCP-1 (placebo + LPS). However, statistically significant suppression of MCP-1 was seen when morphine was co-administered (morphine + LPS). In the absence of LPS, placebo-treated animals showed a low level of MCP-1 expression. As was observed with the morphine + LPS group, MCP-1 expression was further attenuated in the morphine group in the absence of LPS. This trend complements our findings of decreased macrophage infiltration seen in the flow cytometric and immunohistochemical analysis. We at- tribute the decreased macrophage infiltration into PVA sponges to the suppressed chemoattractant expression required for macrophage migration.

Although our findings support the idea that suppression of recruited macrophages toward the site of injury and infection is mediated through a decrease in MCP-1dependent chemoattractant gradient, we were puzzled by the absence of morphine regulation of MIP-2 expression, a potent neutrophil chemoattractant. We speculate that the delay of neutrophil migration following morphine administration maybe at the early phase of neutrophil recruitment and may be a function of $\mathrm{KC}$ expression and independent of MIP-2. To test this hypothesis, we performed a fluorescence screen to measure neutrophil chemotaxis.

\section{Effects of Morphine on Neutrophil Migration Using a Chemotaxis Assay}

Primary naïve neutrophils (Figure 5A) harvested from GFP-transgenic mouse bone marrow were treated with either saline or morphine $(1 \mu \mathrm{mol} / \mathrm{L})$ in vitro for 24 hours and loaded in the top chambers of a high throughput permeable membrane chemotaxis plate. PVA sponge supernatant extracts from day 1, placebo + LPS- and morphine + LPS-treated mice were loaded in the bottom chambers. Migration of saline- and morphine-treated GFP-neutrophils toward placebo- or morphine-treated supernatants were assessed and quantified. There was a robust migration of saline-treated neutrophils toward placebo + LPS sponge supernatant (Figure 5A), however a significant reduction in migration of saline-treated neutrophils was observed when exposed to morphine + LPS supernatant although MIP-2 levels in the supernatant were similar to the placebo + LPS group. Surprisingly, migration of morphine-treated neutrophils toward placebo + LPS supernatant was significantly lower than saline-treated neutrophils. Migration was further reduced when exposed to morphine + LPS supernatant. These data corroborated the in vivo data showing reduced migration of neutrophils into PVA sponges in morphinetreated animals. Furthermore, the failure of migration of morphine-treated neutrophils toward placebo + LPS supernatant, which contained high levels of both $\mathrm{KC}$ and MIP 2 indicate that reduced chemokine levels was not the only mechanism responsible for the reduced migration of neutrophils in morphine-treated animals.

To establish that the migration of neutrophils toward placebo + LPS and morphine + LPS sponge supernatant was independent of MIP-2, sponge supernatant were treated with MIP-2 antibody and added to the bottom chamber and chemotaxis of neutrophils were determined (Figure 5A). Although a reduction in chemotaxis was observed when both placebo + LPS and morphine + LPS supernatant were pretreated with MIP-2 antibody, the decrease in the placebo + LPS supernatant was not as dramatic as the migration observed toward, morphine + LPS supernatant establishing that MIP-2 may not be the principal chemoattractant responsible for the recruitment of neutrophils in our wound model. To determine whether 
A

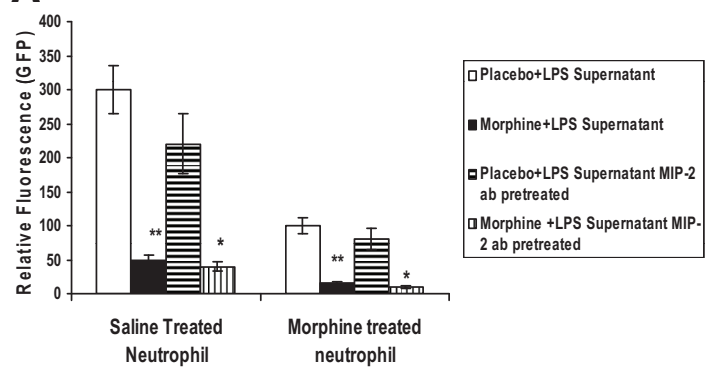

C
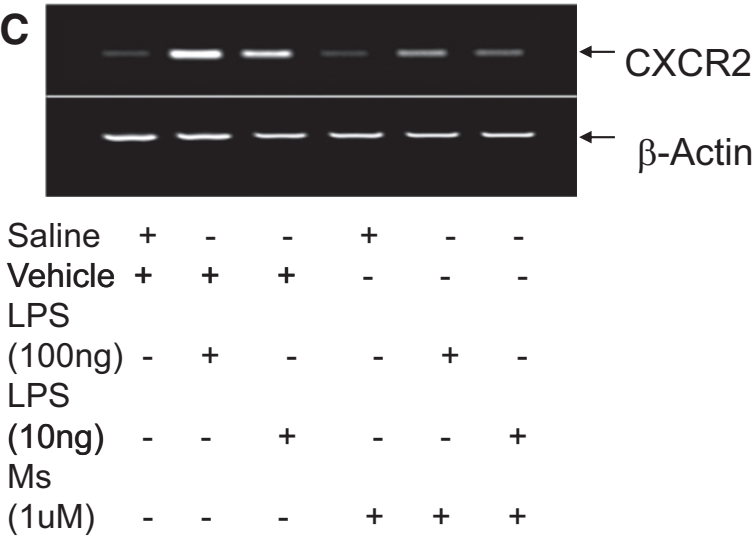

B

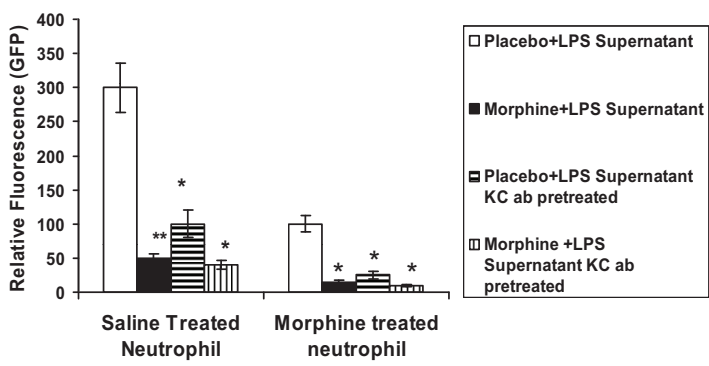

D

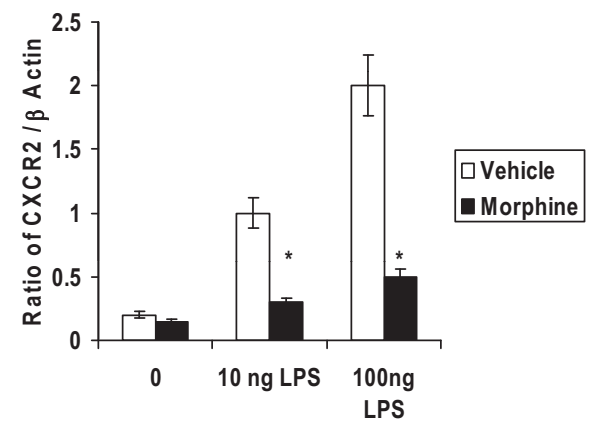

Figure 5. A: Chemotaxis assay of naïve neutrophil migration toward supernatants of PVA sponge extracts. Bone marrow was harvested from GFP-transgenic mice and treated with morphine $(1 \mu \mathrm{mol} / \mathrm{L})$ or saline for 24 hours. $5 \times 10^{6}$ neutrophils were added to top chambers of chemotaxis plates. Supernatants from day 1 PVA sponge extracts were placed in the bottom chambers. Following 1-hour incubation, top chambers were removed, and cells in the bottom chambers were quantified by plate reader using GFP excitation and emission wavelengths (GFP was detected at a 485:520 excitation/emission wavelength). Significantly fewer neutrophils migrated toward the supernatants containing morphine, as compared with the placebo treated group $(P \leq 0.001, n=4)$. In parallel studies, day 1 PVA supernatant was pretreated with either MIP-2 or KC antibody for 12 hours before being placed on the top chamber of the chemotaxis plate. Pretreatment with MIP-2 antibody did not significantly decrease chemotaxis of cells toward the bottom chamber. B: In contrast, pretreatment with KC antibody resulted in a significant decrease in migration of naïve neutrophil toward placeco+LPS supernatant. C and D: Morphine inhibits LPS-induced CXCR2 expression in neutrophils. Bone marrow-derived neutrophils were pretreated with morphine $(1 \mu \mathrm{mol} / \mathrm{L})$ for 24 hours and treated with LPS (10 ng and $100 \mathrm{ng})$ for 2 hours and CXCR2 expression determined using reverse transcription PCR. Results show that morphine treatment resulted in a dramatic decrease in LPS-induced CXCR2 expression levels (D). $\beta$-actin was used as a loading control $\left({ }^{*} P \leq 0.01,{ }^{* *} P \leq 0.001, n=4\right)$. D: Densitometric analysis of four representative PCR gel blots. ( $\left.{ }^{*} P<0.01\right)$.

KC was the principal chemoattractant in the day 1 sponge fluid, sponge supernatant was pretreated with $\mathrm{KC}$ antibody (Figure 5B) and subjected to chemotaxis. Pretreatment of placebo + LPS supernatant with KC antibody resulted in a dramatic decrease in neutrophil recruitment suggesting that $\mathrm{KC}$ may play a dominant role in the early recruitment of neutrophils to the wound site. The delay in neutrophil recruitment in morphine-treated animals may be a consequence of a delay in KC expression.

Since morphine-treated neutrophils showed a dramatic decrease in migration toward placebo + LPS supernatant, we next determined if the decrease in migration of morphine-treated neutrophils toward placebo supernatant was due to a decrease in the MIP-2/KC receptor, CXCR2 (Figure $5 \mathrm{C}$ ). In these studies, bone marrow-derived neutrophils were pretreated with morphine $(1 \mu \mathrm{mol} / \mathrm{L})$ for 24 hours and treated with LPS (10 ng and $100 \mathrm{ng}$ ) for 2 hours and CXCR2 expression was determined using reverse transcription PCR (Figure 5D). As expected LPS treatment resulted in a dose-dependent increase in CXCR2 expression levels. However, morphine pretreatment resulted in a significant decrease in LPS induced CXCR2 expression level. These results suggest that down-regulation of MIP-2/KC receptors following morphine treatment could be a potential mechanism for the observed decreased migration of morphinetreated neutrophils toward a MIP2/KC chemokine gradient. These results also suggest that the delay in neutrophil recruitment following morphine treatment may be due to both a decrease in $\mathrm{KC}$ chemokine during the early phase of wounding and a decrease in CXCR2 expression in neutrophils.

\section{Effect of in Vitro Morphine Treatment on MCP-1 and CCR2 Expression}

To determine whether expression of MCP-1 and its receptor CCR2 in macrophages is modulated by morphine, naïve peritoneal macrophages were pretreated with morphine for 24 hours and then treated with LPS for 12 and 24 hours. MCP-1 and CCR2 expression was determined using reverse transcription PCR. LPS treatment resulted in a time-dependent increase in MCP-1 expression (Figure 6, A and B). However, morphine treatment of macrophages significantly inhibited LPS induced MCP-1 induction supporting our findings observed in vivo. Interestingly, LPS treatment resulted in a time-dependent decrease in CCR2 expression with an inverse relationship to that observed with $\mathrm{MCP}-1$ induction (Fig- 

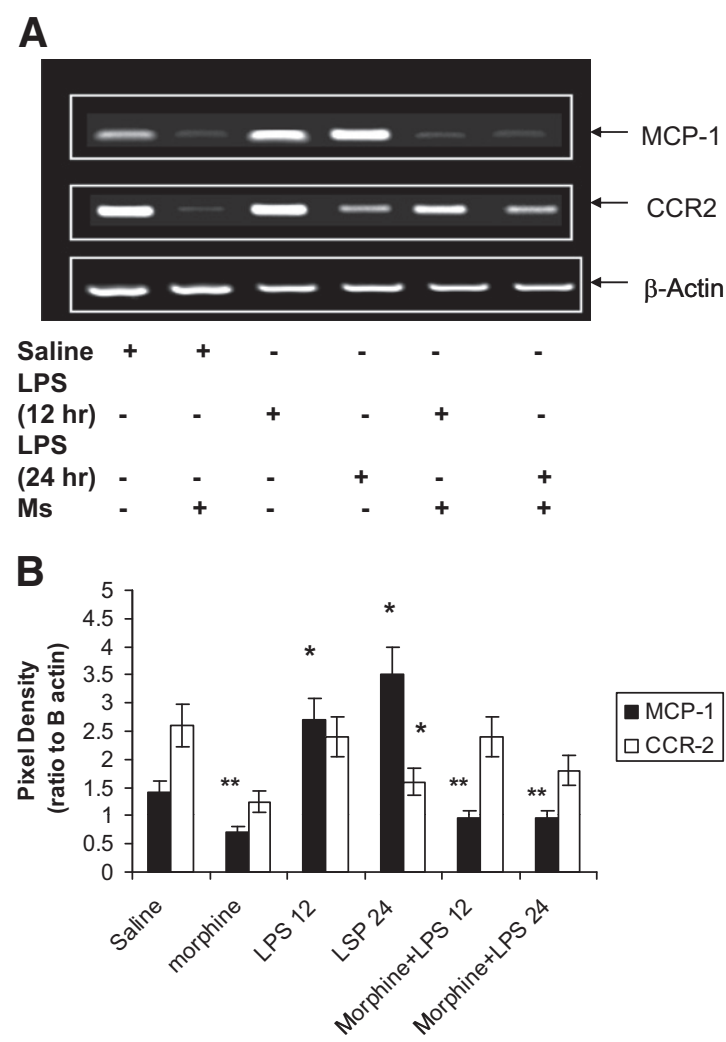

Figure 6. A: Morphine inhibits LPS-induced MCP-1 expression and had no effect on CCR2 expression in macrophages. Naïve peritoneal macrophages were pretreated with either saline or morphine $(1 \mu \mathrm{mol} / \mathrm{L})$ for 24 hours and then treated with LPS for 12 and 24 hours. MCP-1 and CCR2 receptor expression was determined using reverse transcription PCR. LPS treatment resulted in a time-dependent increase in MCP-1 expression. However, morphine treatment of macrophages significantly inhibited LPS-induced MCP-1 induction. Morphine treatment did not result in a decrease in CCR2 expression. B: Densitometric analysis of 4 representative PCR gel blots. $\left({ }^{*} P<0.01\right.$ and ${ }^{* *} P<001$ ).

ure $6, A$ and $B$ ). Morphine treatment did not modulate LPS-induced CCR2 expression.

Taken together, these results suggest that the observed decrease in macrophage recruitment following morphine treatment is a consequence of a decrease in MCP-1 induction. The sustained expression of CCR2 in morphine-treated macrophage may also explain the sustained recruitment of macrophages in the wounds of morphine-treated animals.

\section{Effects of Chronic Morphine on Bacterial Dissemination}

Several groups have associated prolong endotoxin-mediated immune activation to delayed wound healing and septic shock. These studies attribute septicemia to monocyte deactivation, ${ }^{36}$ compromised leukocyte killing activity, ${ }^{37}$ circulating thrombosis, and reduced plasma viscosity. ${ }^{38}$

We next investigated whether the immunosuppressive effects observed following chronic morphine administration would lead to dissemination of bacteria out of wound beds and into the blood stream and other organ compartments. To determine whether chronic morphine treat-

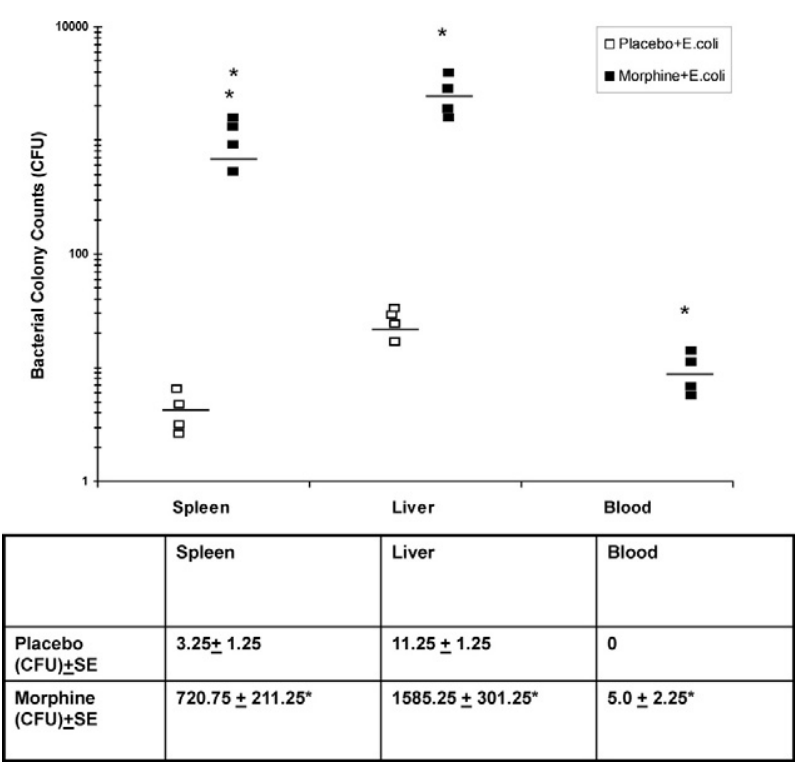

Figure 7. Dissemination of $E$. coli out of wound tissues into blood and organs. PVA sponges containing live bacteria E. coli at 18 colony forming units were implanted into hind limbs of mice administered placebo or morphine. Homogenized spleens, livers, and blood samples were incubated on blood agar plates. Averages of plate colony counts $(n=4)$ were taken. A significant increase in GFP-tagged $E$. coli was seen in the spleen, liver, and blood of morphine-treated groups 7 days following injury ( ${ }^{*} P<0.01, n=4$ ). Adequate clearance of bacteria was found to occur in the blood, spleen, and liver tissues of placebo-treated mice. Horizontal lines represent mean bacterial load. Mean $\pm \mathrm{SE}$ is shown in the table below.

ment results in bacterial dissemination, PVA sponge implants were saturated with live GFP-tagged E. coli (18 colony forming units). Homogenized organ tissues and blood samples taken on day seven post injury, were plated onto blood agar plates and incubated overnight. E. coli GFP-positive colonies were counted. Seven days post-injury, very little $E$. coli dissemination was observed in the spleen and liver and was completely absent in the blood of the placebo-treated group. In contrast, significant number of bacterial colonies grew on blood agar plates from spleen, liver, and blood samples taken from morphine-treated groups (Figure 7). These findings further support our hypothesis that chronic morphine-mediated immunosuppressive effects will lead to detrimental outcomes of delayed wound healing and increased dissemination of bacterial pathogens.

\section{Effects of Chronic Morphine on Angiogenesis}

Macrophages present in inflammatory infiltrate contribute to the angiogenic process by producing a broad array of angiogenic growth factors and cytokines that promote wound remodeling and angiogenesis. To further validate if morphine induced decrease in macrophage infiltration will result in a decrease in angiogenesis, Matrigel admixed with day 4 culture supernatant (obtained from sponges from morphine- and placebo-treated animals) were injected s.c. just above the left shank in animals that were either treated with placebo or morphine pellets. Matrigel plugs were surgically removed on day 7 and snap-frozen. Cryostat sections were fixed in ice-cold acetone and stained with anti-CD31-PE (red images) mono- 


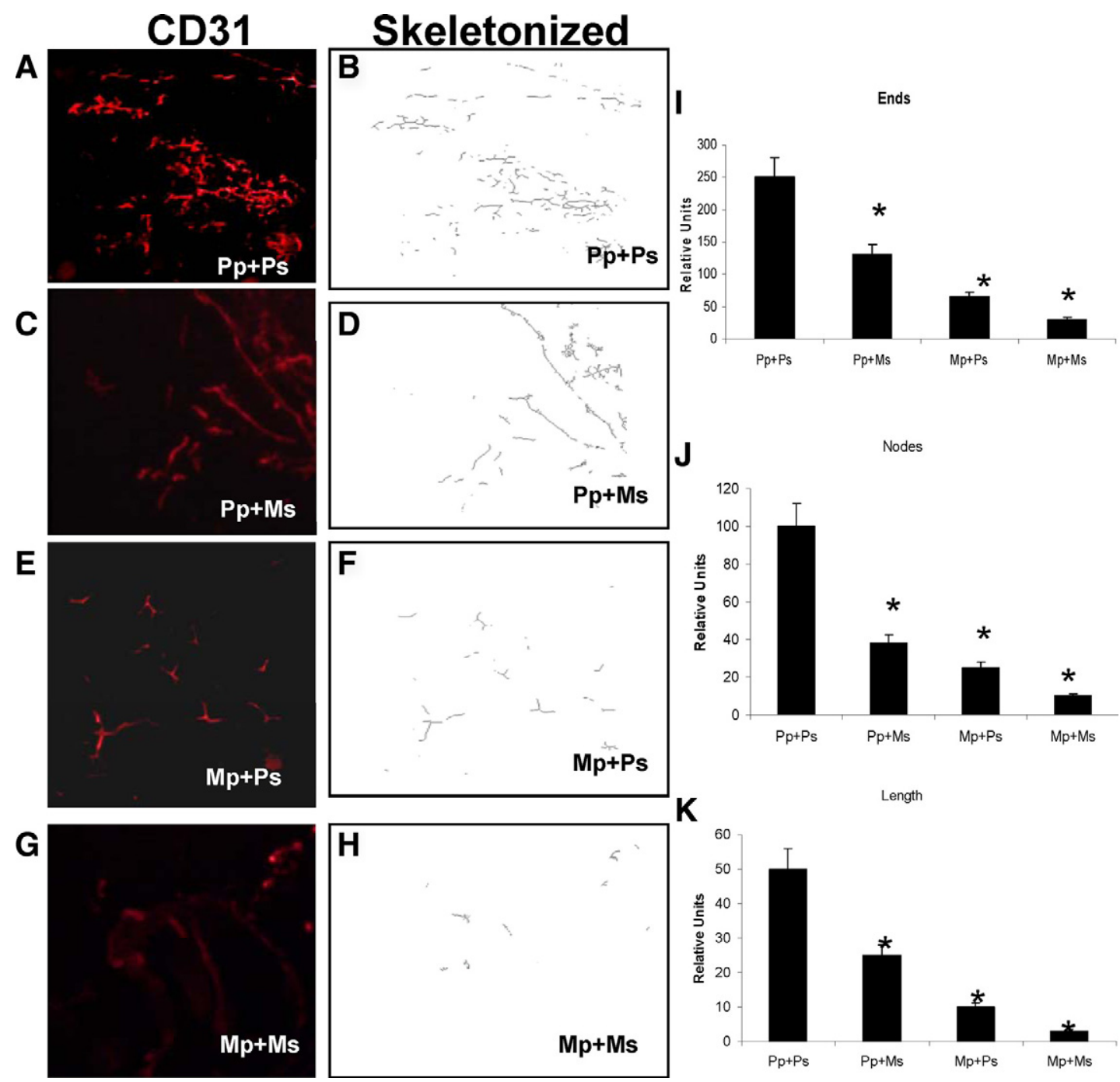

Figure 8. Sponge supernatant from morphine-treated mice attenuated new blood vessel formation in Matrigel plugs. Placebo and morphine PVA sponge supernatants containing Matrigels were injected into the hind limb of placebo or morphine pelleted mice. Matrigel plug was removed at day 7 following euthanasia. Cryostat sections from liquid nitrogen-snap-frozen Matrigel samples were stained with PECAM (CD31) endothelial marker. In the placebo-treated animals that received placebo supernatant $(\mathrm{Pp}+\mathrm{Ps})(\mathbf{A})$ angiogenesis was apparent and readily visible throughout the day 7 Matrigel plugs. Substantial decrease in angiogenesis was observed in the placebo-treated animals that received morphine supernatant $(\mathrm{Pp}+\mathrm{Ms})(\mathbf{C})$. Chronic morphine treatment significantly decreased the formation of blood vessels in the placebo supernatant $(\mathrm{Mp}+\mathrm{Ps})(\mathbf{E})$-containing plugs. The most dramatic decrease in angiogenesis was observed in morphine-treated animals that received morphine sponge supernatant $(\mathrm{Mp}+\mathrm{Ms})(\mathbf{G})$. Panels $\mathbf{B}, \mathbf{D}, \mathbf{F}$, and $\mathbf{H}$ are skeletonized images of panels $\mathbf{A}, \mathbf{C}, \mathbf{E}$, and G. respectively. B: Areas of vessel density in Matrigel plugs were then examined under higher magnification $(\times 20)$ and counted after fluorescent images were binearized for PE-positive pixels, and skeletonized (gray images $\mathbf{B}, \mathbf{D}, \mathbf{F}$, and $\mathbf{H}$ ) using Reindeer Image Analysis tool kit and Adobe PhotoShop. I, J, K: Morphometric analysis of angiogenic events. Quantification of the skeletonized images, shown in Figure 8, B, D, F, and $\mathbf{H}$ was evaluated for angiogenic events [vessel density-ends (I), vessel branch points-nodes $(\mathbf{J})$, and vessel length $(\mathbf{K})$ of new vessels]. Plugs extracted from mice treated with morphine pellets showed a significant reduction in blood vessel density (ends), vessel branching (nodes), and vessel length, when compared with placebo treated groups $\left({ }^{*} P<0.05, n=4\right)$.

clonal antibody at a dilution of 1:100. Angiogenesis in response to placebo- and morphine-treated sponge supernatants was determined using two sections from each plug $(n=5)$ and analyzed for CD31 immunolocalization as described in Figure 2C. Animals that were implanted with a placebo pellet and then implanted with Matrigel plugs admixed with placebo supernatant (Pp + Ps) were highly vascular and showed significant vessel growth (Figure 8, A and B). There was significant decrease in angiogenesis in Matrigel plugs that were resuspended with sponge fluids from morphine-treated animals, even though the host animals were implanted with placebo pellet (Pp + Ms-Figure 8, C and D). A similar decrease was observed in animals that were morphine pelleted but received placebo supernatant (Mp + Ps-Figure 8, E and F). The greatest reduction in angiogenesis was observed in the animals that were morphine-pelleted and received morphine sponge supernatant ( $\mathrm{Mp}+\mathrm{Ms}$-Figure 8, $\mathrm{G}$ and $\mathrm{H}$ ). All three indices of angiogenesis, vessel density (ends Figure $8 \mathrm{I})$, vessel branch point (nodes Figure 8J), and vessel maturation (length-Figure 8K) were significantly decreased in animals that received morphine supernatant and were implanted with morphine pellets. These results indicate that morphine treatment inhibits the host's ability to respond to an angiogenic stimulus contributing to the delayed wound healing observed in morphine-treated animals. 


\section{Discussion}

There are compelling animal data documenting the immunosuppressive effects of opioids and their causal relationship to worsen outcomes during an infectious challenge. ${ }^{39-41}$ Similar observations have also been documented in the drug abuse population, ${ }^{26,42}$ thereby supporting an animal model approach for the study of opioid use and its impact on pathological states.

Very few studies have systematically investigated the consequences of prolonged morphine administration on wound healing events. Our data shows for the first time that morphine's immunosuppressive effects on early innate immune cell recruitment, which is instrumental for bacterial clearance, leads to a net delay in wound healing events. As neutrophils fail to adequately migrate into the site of injury by 24 hours post-injury, less MCP- 1 is produced and secreted, resulting in a lower chemoattractant gradient that is essential for the recruitment of subsequent innate immune populations, including macrophages. Macrophage infiltration was thereby decreased resulting in a decrease in neutrophil clearance and an exacerbated proinflammatory response. Prolonged inflammation, 7 days post-injury, was seen in the gross wound morphologies taken from morphine-administered groups. These wounds presented with pus (indicative of a pronounced presence of neutrophil debris and decreased macrophage recruitment), tissue necrosis, edema, and poor wound integrity. When naltrexone was co-administered to the morphine groups, these modalities were absent and resembled wound morphologies similar to the placebo group. Furthermore, morphine-induced inhibition in wound closure was also abolished in the MORKO mice, establishing the role of the Mu-opioid receptor in morphine-induced modulation of wound closure.

Interestingly, we observed a temporal shift in the secretion of KC and MIP-2 in the PVA sponge following wounding. In the placebo group, $\mathrm{KC}$ expression levels were observed early, and peaked at day 1 following wounding and declined at day 3. MIP-2 levels however, were not significantly elevated at early time points and peaked only at day 3 . The recruitment pattern of neutrophils followed peak KC expression times, implicating KC as a potent chemokine secreted at an early phase during dermal injury for the recruitment of neutrophils. These studies are consistent with the studies reported by Endlich et $\mathrm{al}^{43}$ where a distinct temporal pattern of MIP-2 and $\mathrm{KC}$ chemokine recruitment was observed following surgical injury suggesting distinct regulatory controls. ${ }^{44}$ Similar temporal shifts in KC and MIP-2 expression were also observed in several models of bacterial infection. ${ }^{45,46}$ These studies imply that KC and MIP-2 are differentially modulated depending on the wound environment and the type of insult.

Our study also showed that morphine treatment selectively inhibited early KC synthesis, but had no effect on MIP-2 induction following wounding. Several studies support a MIP-2-independent mechanism for reduced neutrophil migration following injury, especially in an infection model. In an acute alcohol model, even though CXCR2/ MIP2-mediated signaling contributed to reduced infiltra- tion into non-septic wounds, ${ }^{47}$ however under conditions where there is susceptibility to infections such as burn injury, neutrophils failed to enter the tissue despite significantly high levels of MIP-2 chemokines in the wound tissue. ${ }^{47}$ These studies support our conclusion that assigns a relatively reduced role for MIP-2, but a more dominant role for $\mathrm{KC}$ in the recruitment of neutrophils following cutaneous wounding when infection is present.

In wound-healing models, MIP-2 has been shown to be secreted by resident macrophages and dendritic cells at the wound site, whereas $\mathrm{KC}$ is rapidly secreted following injury by damaged epithelial cells. The mechanism for the activation of resident macrophages in the release of chemokines is not clearly understood; however, infection and hypoxia at the wound site have been implicated as potential mechanism by several studies. Studies in support of wounding as a potent stimulus for $\mathrm{KC}$ secretion is provided by several recent reports. ${ }^{48,49}$ These studies identify wound edge keratinocytes as an important source of cytokines in the acute phase following wounding and $\mathrm{KC}$ as the predominant chemokine present at enhanced levels at wound sites following incision. Although our studies show significant decrease in the early expression of $\mathrm{KC}$ following morphine treatment, the cell type responsible for $\mathrm{KC}$ expression was not identified. However, several studies, ${ }^{50-52}$ including ours (unpublished observation), clearly demonstrate the surface expression of mu opioid receptors on keratinocytes. Also, a recent report by Clark et $\mathrm{al}^{48}$ shows significant inhibition of early $\mathrm{KC}$ expression from wound edge keratinocytes following acute morphine treatment. These studies implicate keratinocytes as the potential source of KC synthesis in our model of wound healing and morphine modulation of KC synthesis may be mediated through mu opioid receptors.

When CXCR2 (MIP-2/KC receptor) expression levels were evaluated on morphine-treated neutrophils, a significant decrease in LPS-induced CXCR2 levels was observed. Additional studies using chemotaxis assays showed decreased migration of saline-treated neutrophils toward morphine-treated sponge supernatant, although CXCR2 expression levels were elevated in these cells. Pretreatment of sponge supernatant with MIP-2 antibodies did not significantly alter the migration of neutrophils. However pretreatment of sponge supernatants with $\mathrm{KC}$ antibody resulted in a significant decrease in neutrophil migration in saline-treated neutrophils toward either placebo-treated supernatant or morphine-treated supernatant. Taken together, these results support our conclusion that decreased migration of neutrophils into wound beds following morphine treatment may be a consequence of both a decrease in $\mathrm{KC}$ expression in the wound beds and a decreased expression of CXCR2 on neutrophils.

Morphine treatment resulted in a significant decrease in macrophage recruitment, both in the presence and absence of LPS. A concurrent decrease in MCP-1 levels was also observed when sponge fluids were evaluated for MCP-1 protein levels. Consistent with the in vivo studies, in vitro treatment of macrophages with morphine resulted in a significant decrease in LPS-induced MCP-1 
expression. Interestingly, however, while LPS treatment decreased CCR2 receptor expression levels in a timedependent manner, morphine treatment did not result in any change in CCR2 expression levels. Several reports indicate down-regulation of CCR2 expression as a mechanism for halting sustained recruitment of macrophage in an inflammation model. ${ }^{53}$ Similarly, in our studies we observed a peak recruitment of macrophages at day 3 that decreased progressively and returned to base line levels at day 5 in the placebo group. The decrease in macrophage recruitment can be attributed to a downregulation of CCR2 expression. In contrast, morphine treatment did not alter LPS-induced down-regulation of CCR2 receptor expression. Consistently, we observed a sustained recruitment of macrophages in the morphinetreated animals at times when macrophage levels returned to baseline levels in the placebo group. We hypothesize that morphine modulation of inflammation-induced CCR2 expression may be a potential mechanism for the sustained recruitment of macrophages observed in morphine-treated animals.

In in vivo studies of morphine administration, in the presence of live bacteria, showed stark evidence of the detrimental ramifications that are a result of chronic morphine treatment. Seven days post-injury, when bacterial pathogens were cleared in uncompromised immune animals, significant bacterial dissemination out of the PVA sponge into the blood, spleens, and livers was observed in mice treated with morphine. This finding lends further evidence to the conclusion that chronic morphine effects on early innate immune function have profound impacts on later immune processes that can potentiate secondary infection pathologies such as sepsis.

The clearance of the debris and infectious organisms promotes resolution of inflammatory phase and initiation of the repair phase, which includes formation of granulation, re-epithelialization, and neovascularization. Macrophages play a vital role not only in producing and secreting potent pro-inflammatory factors, but also in their secretion of potent pro-angiogenic factors. With lower macrophage influx, fewer pro-angiogenic factors, including the transcription factor hypoxia inducible factor- $1 \alpha$ and growth factor VEGF, are produced and secreted by these cells. In the absence of these factors, the second arm of healing cannot proceed resulting in significantly delayed wound repair. Our studies show a prolonged inflammatory phase in morphine-treated animals with a significant decrease in angiogenesis. We hypothesize that the lack of resolution of the inflammatory phase in morphine-treated animals delays the initiation of the repair phase.

We have previously shown that morphine treatment significantly inhibits hypoxia-induced hypoxia inducible factor- $1 \alpha$ expression with concurrent decrease in VEGF-A synthesis and VEGF-induced angiogenesis in a myocardial ischemic model, ${ }^{54,55}$ and more recently, in a tumor-induced angiogenesis model (unpublished data). We speculate that the reduction in angiogenesis observed following morphine treatment in our wound healing model may be due to a combination of a decrease in VEGF synthesis and reduced recruitment of macrophages. Furthermore, a recent study by Lam et al ${ }^{56}$ shows impaired mobilization of endothelial progenitor cells following high-dose morphine treatment with a concurrent decrease in angiogenesis. Impaired recruitment of progenitor endothelial cells following wounding as an additional potential mechanism for compromised angiogenesis following morphine treatment cannot be discounted.

These studies provide an enhanced understanding of the effects of chronic morphine administration on wound healing processes. Scientific contributions of these studies provide an in vivo tool by which further mechanistic experiments can be performed to address why clinically, heroin-addicted patients often present with infected nonhealing wounds. Understanding these underlying mechanisms affords improved treatment options not only for chronic morphine users and abusers, but can also have translational implications for immuno-compromised populations such as the elderly ${ }^{57}$ or those who are chronically stressed. ${ }^{58}$

\section{References}

1. Abbadie C, Pan YX, Pasternak GW: Immunohistochemical study of the expression of exon11-containing mu opioid receptor variants in mouse brain. Neuroscience 2004, 127:419-430

2. Peckys D, Landwehrmeyer GB: Expression of mu, kappa, and delta opioid receptor messenger RNA in the human CNS: a 33P in situ hybridization study. Neuroscience 1999, 88(4):1093-135

3. Zastawny RL, George SR, Nguyen T, Cheng R, Tsatsos J, BrionesUrbina R, O'Dowd BF: Cloning, characterization, and distribution of a mu-opioid receptor in rat brain. J Neurochem 1994, 62:2099-2105

4. Börner C, Stumm R, Höllt V, Kraus J: Comparative analysis of muopioid receptor expression in immune and neuronal cells. J Neuroimmunol 2007, 188:56-63

5. Chuang TK, Killam KF Jr., Chuang LF, Kung HF, Sheng WS, Chao CC, Yu L, Chuang RY: Mu opioid receptor gene expression in immune cells. Biochem Biophys Res Commun 1995, 216:922-930

6. Bueno L, Fioramonti J: Action of opiates on gastrointestinal function Baillieres Clin Gastroenterol 1988, 2:123-139.

7. Page GG: Immunologic effects of opioids in the presence or absence of pain. J Pain Symptom Manage 2005, 29:S25-S31

8. Sacerdote P, Bianchi M, Gaspani L, Manfredi B, Maucione A, Terno G, Ammatuna M, Panerai AE: The effects of tramadol and morphine on immune responses and pain after surgery in cancer patients. Anesth Analg 2000, 90:1411-1414

9. Stein C, Machelska H, Schafer M: Peripheral analgesic and antiinflammatory effects of opioids. Z Rheumatol 2001, 60:416-424

10. Vallejo R, de Leon-Casasola O, Benyamin R: Opioid therapy and immunosuppression: a review. Am J Ther 2004, 11:354-365

11. Willette RN, Sapru HN: Peripheral versus central cardiorespiratory effects of morphine. Neuropharmacology 1982, 21:1019-1026

12. Diegelmann RF, Evans MC: Wound healing: an overview of acute, fibrotic and delayed healing. Front Biosci 2004, 9:283-289

13. DiPietro LA: Wound healing: the role of the macrophage and other immune cells. Shock 1995, 4:233-240

14. Frantz S, Vincent KA, Feron O, Kelly RA: Innate immunity and angiogenesis. Circ Res 2005, 96:15-26

15. Glaser R, Kiecolt-Glaser JK: Stress-induced immune dysfunction: implications for health. Nat Rev Immunol 2005, 5:243-251

16. Moore K: Cell biology of chronic wounds: the role of inflammation. J Wound Care 1999, 8:345-348

17. Naldini A, Carraro F: Role of inflammatory mediators in angiogenesis. Curr Drug Targets Inflamm Allergy 2005, 4:3-8

18. Olah ME, Caldwell CC: Adenosine receptors and mammalian toll-like receptors: synergism in macrophages. Mol Interv 2003, 3:370-374

19. Tidball JG: Inflammatory processes in muscle injury and repair. Am J Physiol Regul Integr Comp Physiol 2005, 288:R345-R53

20. Whelan C, Stewart J, Schwartz BF: Mechanics of wound healing and 
importance of vacuum assisted closure in urology. J Urol 2005, 173:1463-1470

21. Park JE, Barbul A: Understanding the role of immune regulation in wound healing. Am J Surg 2004, 187:11S-16S

22. Lee $R H$, Efron $D$, Tantry $U$, Barbul A: Nitric oxide in the healing wound: a time-course study. J Surg Res 2001, 101:104-108

23. Ley K, Laudanna C, Cybulsky MI, Nourshargh S: Getting to the site of inflammation: the leukocyte adhesion cascade updated. Nat Rev Immunol 2007, 7:678-689

24. Canturk NZ, N Esen, B Vural, Z Canturk, G Kirkali, G Oktay, S Solakoglu: The relationship between neutrophils and incisional wound healing. Skin Pharmacol App Skin Physiol 2001, 14:108-116

25. Tsirogianni AK, Moutsopoulos NM, Moutsopoulos HM: Wound healing: Immunological aspects. Injury 2006, 37:S5-S12

26. Alonzo NC, Bayer BM: Opioids, immunology, and host defenses of intravenous drug abusers. Infect Dis Clin North Am 2002, 16:553-569

27. Dighe SV, Madia PA, Sirohi S, Yoburn BC: Continuous morphine produces more tolerance than intermittent or acute treatment. Pharmacol Biochem Behav 2009, 92:537-542

28. Chen TC, Cheng YY, Sun WZ, Shyu BC: Differential regulation of morphine antinociceptive effects by endogenous enkephalinergic system in the forebrain of mice. Mol Pain 2008, 4:41

29. Osikowicz M, Mika J, Makuch W, Przewlocka B: Glutamate receptor ligands attenuate allodynia and hyperalgesia and potentiate morphine effects in a mouse model of neuropathic pain. Pain 2008, 139:117-126

30. Rook EJ, van Ree JM, van den Brink W, Hillebrand MJ, Huitema AD, Hendriks VM, Beijnen JH: Pharmacokinetics and pharmacodynamics of high doses of pharmaceutically prepared heroin, by intravenous or by inhalation route in opioid-dependent patients. Basic Clin Pharmacol Toxicol 2006, 98:86-96

31. Tiseo PJ, Thaler HT, Lapin J, Inturrisi CE, Portenoy RK, Foley KM: Morphine-6-glucuronide concentrations and opioid-related side effects: a survey in cancer patients. Pain 1995, 61:47-54

32. Wang J, Barke RA, Charboneau R, Roy S: Morphine impairs host innate immune response and increases susceptibility to Streptococcus pneumoniae. J Immunol 2005, 174:426-434

33. Deshmukh HS, Hamburger JB, Ahn SH, McCafferty DG, Yang SR, Fowler VG Jr: Critical role of NOD2 in regulating the immune response to Staphylococcus aureus. Infect Immun 2009, 77:1376-1382

34. Wild R, Dhanabal M, Olson TA, Ramakrishnan S: Inhibition of angiogenesis and tumour growth by VEGF121-toxin conjugate: differential effect on proliferating endothelial cells. Br J Cancer 2002, 83:1077-1083

35. Donovan J, Brown P: Blood collection. Curr Protoc Immunol 2006, Chapter 1:Unit 1.7

36. Kox WJ, Volk T, Kox SN, Volk HD: Immunomodulatory therapies in sepsis. Intensive Care Med 2000, 26:S124-S128

37. Rico RM, Ripamonti R, Burns AL, Gamelli RL, DiPietro LA: The effect of sepsis on wound healing. J Surg Res 2002, 102:193-197

38. Hardaway RM: A review of septic shock. Am Surg 2000, 66:22-29

39. Peterson PK, Sharp BM, Gekker G, Portoghese PS, Sannerud K, Balfour HH Jr: Morphine promotes the growth of HIV-1 in human peripheral blood mononuclear cell cocultures. AIDS 1990, 4:869-873

40. Roy S, Loh HH: Effects of opioids on the immune system. Neurochem Res 1996, 21:1375-1386

41. Roy S, Wang J, Kelschenbach J, Koodie L, Martin J: Modulation of immune function by morphine: implications for susceptibility to infection. J Neuroimmune Pharmacol 2006, 1:77-89

42. Kreek MJ: Immune function in heroin addicts and former heroin addicts in treatment: pre- and post-AIDS epidemic. NIDA Res Monogr 1990, 96:192-219
43. Endlich B, Armstrong D, Brodsky J, Novotny M, Hamilton TA: Distinct temporal patterns of macrophage-inflammatory protein-2 and $\mathrm{KC}$ chemokine gene expression in surgical injury. J Immunol 2002, 168: 3586-3594

44. Mehrad B, Strieter RM, Moore TA, Tsai WC, Lira SA, Standiford TJ: CXCR-2 ligands are necessary components of neutrophil mediated host defense in invasive pulmonary aspergillosis. J Immunol 1999, 163:6086-6094

45. Lentsch AB, Yoshidome J, Cheadle WG, Miller FN, Edwards MJ: Chemokine involvement in hepatic ischemia/reperfusion injury in mice:roles for macrophage inflammatory protein-2 and Kupffer cells. Hepatology 1998 27:507-512

46. Tateda K, Moore TA, Newstead MW, Tsai WC, Zeng X, Deng JC, Chen G, Reddy R, Yamaguchi K, Standiford TJ: Chemokine dependent neutrophil recruitment in a murine model of Legionella pneumonia: potential role of neutrophils as immunoregulatory cells. Infect Immun 2001, 69:2017-2024

47. Fitzgerald DJ, Radek KA, Chaar M, Faunce DE, DiPietro LA, Kovacs EJ Effects of acute ethanol exposure on the early inflammatory response after excisional injury. Alcohol Clin Exp Res 2007, 31:317-323

48. Clark JD, Shi X, Li X, Qiao Y, Liang D, Angst MS, Yeomans DC: Morphine reduces local cytokine expression and neutrophil infiltration after incision. Mol Pain 2007, 3:28

49. Liang D, Shi X, Qiao Y, Angst MS, Yeomans DC, Clark JD: Chronic morphine administration enhances nociceptive sensitivity and local cytokine production after incision. Mol Pain 2008, Feb 22;4:7

50. Cheng B, Liu HW, Fu XB, Sheng ZY, Li JF: Coexistence and upregulation of three types of opioid receptors, mu, delta and kappa, in human hypertrophic scars. Br J Dermatol 2008, 158:713-720

51. Bigliardi-Qi M, Sumanovski LT, Büchner S, Rufli T, Bigliardi PL: Muopiate receptor and Beta-endorphin expression in nerve endings and keratinocytes in human skin. Dermatology 2004, 209:183-189

52. Bigliardi PL, Büchner S, Rufli T, Bigliardi-Qi M: Specific stimulation of migration of human keratinocytes by mu-opiate receptor agonists. J Recept Signal Transduct Res 2002, 22:191-199

53. Maus UA, Wellmann S, Hampl C, Kuziel WA, Srivastava M, Mack M, Everhart MB, Blackwell TS, Christman JW, Schlöndorff D, Bohle RM, Seeger W, Lohmeyer J. CCR2-positive monocytes recruited to inflamed lungs downregulate local CCL2 chemokine levels. Am J Physiol Lung Cell Mol Physiol 2005, 288:L350-L358

54. Balasubramanian S, Ramakrishnan S, Charboneau R, Wang J, Barke RA, Roy S: Morphine sulfate inhibits hypoxia-induced vascular endothelial growth factor expression in endothelial cells and cardiac myocytes. J Mol Cell Cardiol 2001, 33:2179-2187

55. Roy S, Balasubramanian S, Wang J, Chandrashekhar Y, Charboneau $\mathrm{R}$, Barke R: Morphine inhibits VEGF expression in myocardial ischemia. Surgery 2003, 134:336-344

56. Lam CF, Chang PJ, Huang YS, Sung YH, Huang CC, Lin MW, Liu YC, Tsai YC: Prolonged use of high-dose morphine impairs angiogenesis and mobilization of endothelial progenitor cells in mice. Anesth Analg 2008, 107:686-692

57. Pergolizzi J, Boger RH, Budd K, Dahan A, Erdine S, Hans G, Kress HG, Langford R, Likar R, Raffa RB, Sacerdote P: Opioids and the management of chronic severe pain in the elderly: Consensus statement of an international expert panel with focus on the six clinically most often used world health organization step III opioids (buprenorphine, fentanyl, hydromorphone, methadone, morphine, oxycodone). Pain Pract 2008, Jul-Aug; 8(4):287-313

58. Ballard KA, Pellegrino TC, Alonzo NC, Nugent AL, Bayer BM: Enhanced immune sensitivity to stress following chronic morphine exposure. J Neuroimmune Pharmacol 2006, 1:106-115 